BNL-111779-2016-JA

\title{
Direct measurement of the tunable electronic structure of bilayer MoS2 by interlayer twist
}

Po-Chun Yeh, Wencan Jin, Nader Zaki, Jens Kunstmann, Daniel Chenet, Ghidewon Arefe, Jerzy T. Sadowski, Jerry I. Dadap, Peter Sutter, James Hone, and Richard M. Osgood, Jr.

Submitted to Nano Letters

January 2016

Center For Functional Nanomaterials

Brookhaven National Laboratory

\author{
U.S. Department of Energy \\ USDOE Office of Science (SC), \\ Basic Energy Sciences (BES) (SC-22)
}

Notice: This manuscript has been authored by employees of Brookhaven Science Associates, LLC under Contract No. DE- SC0012704 with the U.S. Department of Energy. The publisher by accepting the manuscript for publication acknowledges that the United States Government retains a non-exclusive, paid-up, irrevocable, world-wide license to publish or reproduce the published form of this manuscript, or allow others to do so, for United States Government purposes. 


\section{DISCLAIMER}

This report was prepared as an account of work sponsored by an agency of the United States Government. Neither the United States Government nor any agency thereof, nor any of their employees, nor any of their contractors, subcontractors, or their employees, makes any warranty, express or implied, or assumes any legal liability or responsibility for the accuracy, completeness, or any third party's use or the results of such use of any information, apparatus, product, or process disclosed, or represents that its use would not infringe privately owned rights. Reference herein to any specific commercial product, process, or service by trade name, trademark, manufacturer, or otherwise, does not necessarily constitute or imply its endorsement, recommendation, or favoring by the United States Government or any agency thereof or its contractors or subcontractors. The views and opinions of authors expressed herein do not necessarily state or reflect those of the United States Government or any agency thereof. 


\title{
Direct measurement of the tunable electronic structure of bilayer $\mathrm{MoS}_{2}$ by interlayer twist
}

\author{
Po-Chun Yeh, ${ }^{1}$ Wencan Jin, ${ }^{2}$ Nader Zaki, ${ }^{2}$ Jens Kunstmann ${ }^{3,4}$, Daniel Chenet ${ }^{5}$, Ghidewon Arefe ${ }^{5}$, Jerzy T. Sadowski, ${ }^{6}$ Jerry I. \\ Dadap, ${ }^{2}$ Peter Sutter, ${ }^{7}$ James Hone ${ }^{5}$, and Richard M. Osgood, Jr. ${ }^{1,2, *}$ \\ ${ }^{1}$ Department of Electrical Engineering, Columbia University, New York, New York 10027, USA \\ 2 Department of Applied Physics and Applied Mathematics, Columbia University, New York, New York 10027, USA \\ ${ }^{3}$ Department of Chemistry, Columbia University, New York, New York 10027, USA \\ ${ }^{4}$ Theoretical Chemistry, TU Dresden, 01062 Dresden, Germany \\ ${ }^{5}$ Department of Mechanical Engineering, Columbia University, New York, New York 10027, USA \\ ${ }^{6}$ Center for Functional Nanomaterials, Brookhaven National Laboratory, Upton, New York 11973, USA \\ ${ }^{7}$ Department of Electrical and Computer Engineering, University of Nebraska-Lincoln, Lincoln, Nebraska 68588, USA
}

\begin{abstract}
Keywords: Stacked van der Waals Structures, Photoemission, Twisted van der Waals Materials, Spectromicroscopy, Low Energy Electron Microscopy (LEEM), MoS $_{2}$
\end{abstract}

\section{Abstract}

Using angle-resolved photoemission on micrometer-scale sample areas, we directly measure the interlayer twist angle-dependent electronic band structure of bilayer molybdenum-disulfide $\left(\mathrm{MoS}_{2}\right)$. Our measurements, performed on arbitrarily stacked bilayer $\mathrm{MoS}_{2}$ flakes prepared by chemical vapor deposition, provide direct evidence for a downshift of the quasiparticle energy of the valence-band at the

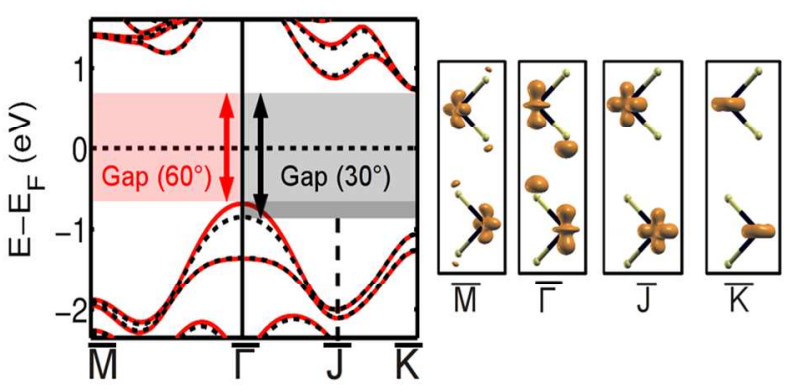
Brillouin zone center ( $\bar{\Gamma}$ point) with the interlayer twist angle, up to a maximum of $120 \mathrm{meV}$ at a twist angle of $\sim 40^{\circ}$. Our direct measurements of the valence band structure enable the extraction of the hole effective mass as a function of the interlayer twist angle. While our results at $\bar{\Gamma}$ agree with recently published photoluminescence data, our measurements of the quasiparticle spectrum over the full 2D Brillouin zone reveal a richer and more complicated change in the electronic structure than previously theoretically predicted. The electronic structure measurements reported here, including the evolution of the effective mass with twist-angle, provide new insight into the physics of twisted transition-metal dichalcogenide bilayers and serve as a guide for the practical design of $\mathrm{MoS}_{2}$ optoelectronic and spin-/valley-tronic devices.

Van der Waals layered materials, especially the transition-metal dichalcogenides (TMDs), can be prepared as atomically thin semiconductors ${ }^{1}$ with high-quality homo- or hetero-junction interfaces. These interfaces can be formed without the restrictions faced by conventional 3D semiconductors in terms of lattice matching or interlayer crystallographic alignment. The utilization of layered materials opens up potential applications for bandgap engineering by using $\operatorname{strain}^{2}$, stacking of layers ${ }^{3,4}$, or building of heterojunctions ${ }^{5,6}$. For TMDs such as $\mathrm{MoS}_{2}, \mathrm{MoSe}_{2}, \mathrm{WS}_{2}$, and WSe $\mathrm{W}_{2}$, the electrical, optical ${ }^{1,7}$, and vibrational properties ${ }^{8}$ are also known to be significantly dependent on interlayer coupling. One of the well-known consequences of interlayer coupling in TMDs is the direct-to-indirect bandgap transition from monolayer to multilayer films. The size of the indirect band gap has also been predicted 
to vary with both the number of layers and the interlayer distance ${ }^{8}$, due to associated changes in interlayer electronic coupling. To date, however, experimental efforts directed toward understanding the interlayer interaction in TMDs, via direct measurements of the electronic band structure, have only been conducted for the case of crystallographically-aligned layers, as found in samples exfoliated from bulk materials $1,7,8,9,10$. Recently, photoluminescence $(P L)^{11,12,13,14}$ and density functional theory $(\mathrm{DFT})^{11,12,13,15,16}$ studies on arbitrarily-aligned bilayer $\mathrm{MoS}_{2}$ flakes (with variable interlayer twist angle) have been reported. In PL measurements, the bilayer $\mathrm{MoS}_{2}$ flakes are prepared by stacking two chemical-vapor-deposition (CVD) prepared monolayer $\mathrm{MoS}_{2}$ flakes.

Based on these recent PL results and Raman measurements of the characteristic phonon modes $\left(E_{2 g}\right.$ and $\left.A_{1 g}\right)$ for twisted-bilayer $\operatorname{MoS}_{2}{ }^{11,12,13,14}$, one can conclude that (1) the interlayer coupling has a global maximum for 0 -degree twist angle, (2) the interlayer coupling of bilayer $\mathrm{MoS}_{2}$ has a local maximum for 60-degree twist angle (bilayer $\mathrm{MoS}_{2}$ per se), and (3) the interlayer coupling is at a minimum when the twist angle lies between 30-40 degrees. Density functional theory studies attribute these results to a twist-angle-dependent change of the layer separation, which consequently determines the degree of energy splitting of the highest occupied states around $\bar{\Gamma}$. The extent of this energy splitting is reflected indirectly in the evolution of the relative energy difference between the photoluminescence $\bar{\Gamma}-\overline{\mathrm{K}}^{*}$ and $\overline{\mathrm{K}}-\overline{\mathrm{K}}^{*}$ transitions, where $\overline{\mathrm{K}}$ and $\overline{\mathrm{K}}^{*}$ denote the highest occupied valence band and the lowest unoccupied conduction band states at the high symmetry point $K$,

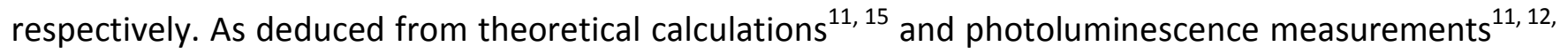
13,14 , the evolution of the uppermost valence band and the lowest conduction band (or conduction band minimum, CBM) with respect to interlayer twist in bilayer $\mathrm{MoS}_{2}$ changes the valence band maximum (VBM) at $\bar{\Gamma}$, while leaving the energy gap at $\overline{\mathrm{K}}$ almost intact (the direct gap changes by $\leq 80$ meV between $0^{\circ}$ and $60^{\circ}$ twist angle ${ }^{11}$ ). In light of these recent reports, there is a pressing need to examine these findings using a more direct probe, and thus to verify the current theoretical predictions via direct experimental measurements of the energy-momentum dispersion, which is not accessible through photoluminescence studies. Furthermore, given the current intense interest in the field to the fabrication and electronic engineering of heterostructures composed of two-dimensional/monolayer materials, it is important to characterize the electronic structure via a direct band structure probing technique, such as angle resolved photoemission (ARPES).

In this paper, we directly measure the energy-momentum-dispersion of CVD-grown and drytransferred twisted bilayer $\mathrm{MoS}_{2}\left(\mathrm{~TB}-\mathrm{MoS}_{2}\right.$ ) for several twist angles ranging from $0^{\circ}$ to $60^{\circ}$ using microspot angle-resolved photoemission spectroscopy ( $\mu$-ARPES). By utilizing bright-field (BF) low-energy electron microscopy (LEEM), we locate twisted bilayer regions of interest, and determine their relative twist angle and their region boundaries by in-situ micro-spot low-energy electron diffraction ( $\mu$-LEED) and dark-field (DF) LEEM imaging. Our $\mu$-ARPES measurements over the entire surface-Brillouin zone reveal that the valence band maximum at $\bar{\Gamma}$ is indeed the highest occupied state for all twist angles, affirming the indirect nature of the bilayer $\mathrm{MoS}_{2}$ bandgap, irrespective of twist angle, as suggested on the basis of photoluminescence spectroscopy ${ }^{11,12,13}$. We directly quantify the energy difference between the high symmetry points at $\bar{\Gamma}$ and $\bar{K}$ in the valence basnd, which is a function of twist angle, 
and observe the same trend reported in the above-mentioned photoluminescence and Raman studies. We confirm that this trend is a result of the energy shifting of the topmost occupied state at $\bar{\Gamma}$, which was previously predicted by DFT calculations (in part in Ref. 11-15 and associated supplemental materials). While our results at $\bar{\Gamma}$ agree with recently published photoluminescence data, our measurements of the quasiparticle spectrum over the full 2D Brillouin zone reveal a richer and more complicated twist angle dependence of the electronic structure than is captured by DFT calculations of isolated bilayers. For example, our measurements over the entire 2D Brillouin zone allows us to determine variations of the hole effective mass at $\bar{K}$ as a function of twist angle.
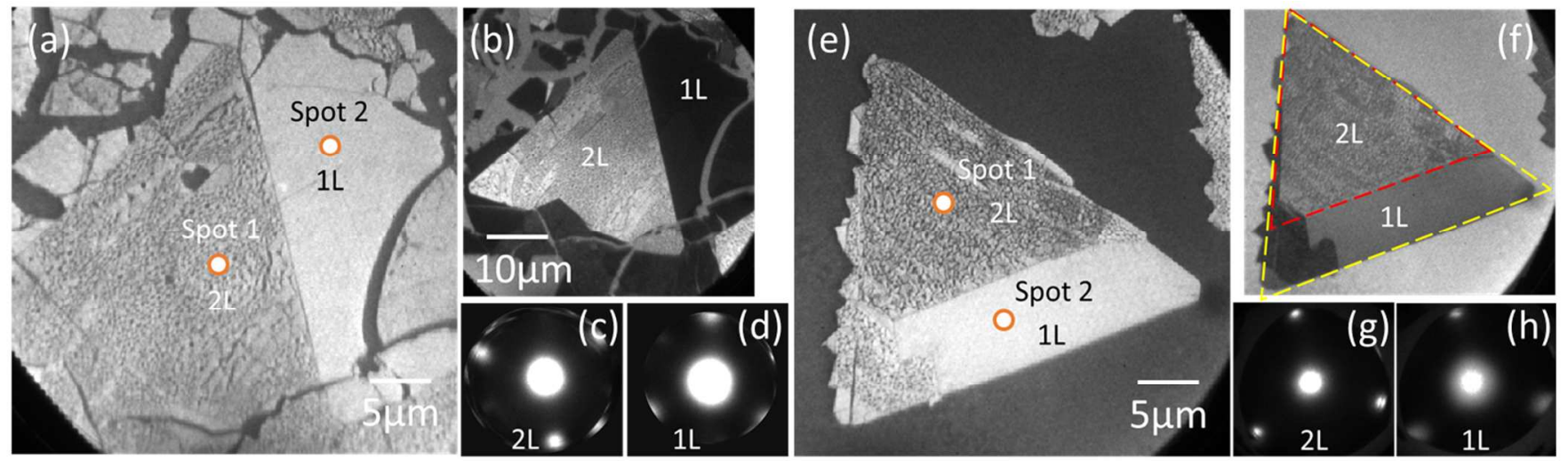

Fig. 1 Bright field (BF) and dark field (DF) LEEM images of monolayer/twisted bilayer $\mathrm{MoS}_{2}$ flakes with twist angles of $47^{\circ}((\mathrm{a}),(\mathrm{b}))$ and $\sim 0^{\circ}((\mathrm{e}),(\mathrm{f}))$, respectively. The CVD growth process yields both isolated islands and connected patches of $\mathrm{MoS}_{2}$. Both were used in our experiment. The markers in (a) and (e) indicate the location of measurements used to determine the twist angle. In (a), spot 1 is located on the bilayer (with triangular upper flake) and spot 2 lies on the larger bottom flake that extends beyond the top flake. (b) DF-LEEM imaging of (a) using a LEED spot originating from the top layer shows a bright bilayer and a dark monolayer section, confirming a non-zero twist angle between the two flakes (spot 1 was used in the DF-LEEM). An analogous placement of measurement spots was chosen in (e), where both the top and bottom flakes are triangular. Since their twist angle is nearly zero, there is minimal contrast in their DF-LEEM image (f). (c) and (d), (g) and (h) are LEED patterns of the bilayer and monolayer segments of flake (a) and (e), respectively showing the same orientation of the diffraction spots. The electron energies used here were (a) $3.5 \mathrm{eV}$, (b) $40 \mathrm{eV}$, (c)-(d) $40 \mathrm{eV}$, (e) $4.6 \mathrm{eV}$, (f) $36.4 \mathrm{eV}$, (g)-(h) $40 \mathrm{eV}$.

Sample quality (see also Supplementary Information) and crystal orientation (i.e., interlayer twist) of our TB-MoS 2 samples were examined using both BF- and DF-LEEM and $\mu$-LEED (Fig. 1). DFLEEM using LEED spots originating from different $\mathrm{MoS}_{2}$ layers shows clear contrast between $\mathrm{ML} \mathrm{MoS}_{2}$ flakes of different crystal orientations (Fig. 1(b) and 1(f)), which makes it preferable over BF-LEEM to identify twisted bilayers (Fig. 1(a) and 1(e)). DF-LEEM also allows us to identify the boundary of a region of interest. Note that in Fig. 1(b), for the case of the $47^{\circ}$ twist angle, the DF-LEEM image shows a distinct contrast between the top (bilayer) and the bottom (exposed monolayer). As for Fig. 1(f), in the case of the $\sim 0^{\circ}$ twist angle, the DF-LEEM image shows almost no contrast between the top (red line) and bottom (yellow line) layer, as expected for identically oriented layers. The corresponding electron diffraction patterns (at a primary electron energy of $40 \mathrm{eV}$ ) of the top and bottom layer of the 47- 
degree and 0-degree TB-MoS 2 are shown in Fig. $1(\mathrm{c}) \&(\mathrm{~d})$ and $1(\mathrm{~g}) \&(\mathrm{~h})$, respectively. At this energy, the LEED patterns clearly show a 3-fold symmetry, and can be used to identify crystal orientation. Note that the LEED pattern of TB-MoS 2 at this energy primarily reflects the orientation of the topmost layer due to the limited electron penetration depth, as similarly found for graphene ${ }^{17,18}$. Furthermore, we note the absence of any additional/satellite diffraction spots, which indicates a lack of a significant super-lattice (moiré) potential on the bilayers. Note that the non-uniform intensity of the top layer has been attributed to roughness induced during the transfer process (see Supplementary Material of ref. [10]).
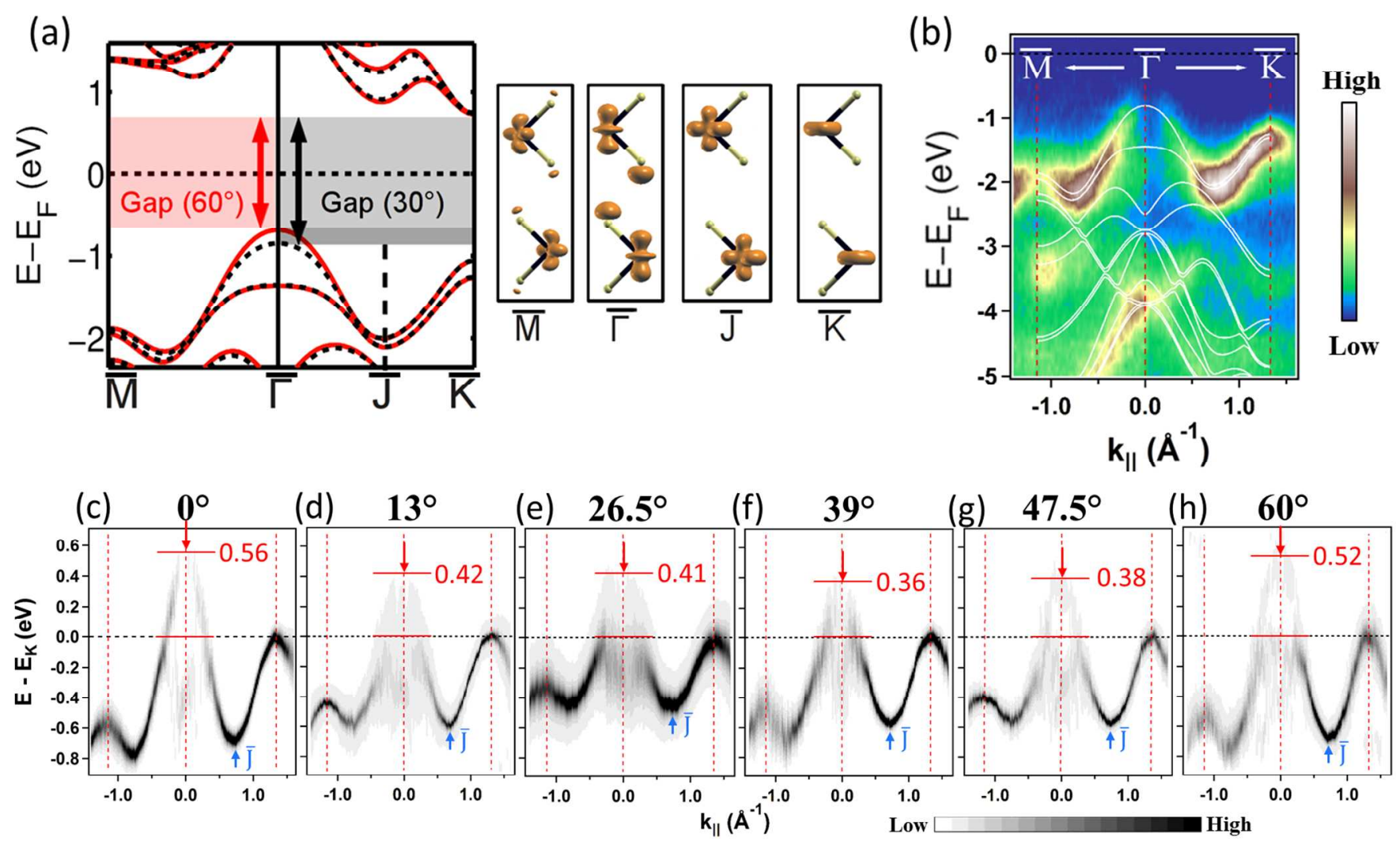

Fig. 2 (a) DFT-calculated band structure of a $60^{\circ}$-twisted (normal bilayer) TB-MoS 2 at interlayer spacings corresponding to $60^{\circ}$ (red solid lines) and $30^{\circ}$ (black dashed lines). The spacing is larger for $\theta=30^{\circ}$ compared to that for $\theta=60^{\circ}$ by $\sim 0.3 \AA$. The energy of the top-most valence band at $\bar{\Gamma}$ varies strongly with the change of the interlayer spacing, opening the band gap this way, while the, $\overline{\mathrm{M}}, \overline{\mathrm{J}}$ and $\overline{\mathrm{K}}$ points are not strongly influenced. The orbital character of selected states are marked. (b) $\mu$-ARPES measurement of bilayer $\mathrm{MoS}_{2}$ (with twist angle $\theta=0^{\circ}$ ), shown as a cut along $\overline{\mathrm{M}}-\bar{\Gamma}-\overline{\mathrm{K}}$. The white continuous lines correspond to the DFT-calculated band structure. For $\theta=0^{\circ}$ and $60^{\circ}$ we obtain excellent agreement between ARPES and DFT. Plots of ARPES measurements for additional twist angles can be found in Fig. S3 in the supplementary section. (c)-(h) Second-derivative-filtered topmost valence band as shown by $\mu$-ARPES measurements (cut along $\overline{\mathrm{M}}-\bar{\Gamma}-\overline{\mathrm{K}}$ ) for TB-MoS 2 with twist angles (c) $0^{\circ}$, (d) $13.5^{\circ}$, (e) $26.5^{\circ}$, (f) $39^{\circ}$, (g) $47.5^{\circ}$, and (h) $60^{\circ}$. Red dashed lines indicate the positions of $\overline{\mathrm{M}}, \bar{\Gamma}$, and $\overline{\mathrm{K}}$ from left to right, respectively. We denote the local minimum located between $\bar{\Gamma}$ and $\overline{\mathrm{K}}$ as the $\bar{J}$ point, marked by a blue arrow. The number (red) overlaid on each band reports the average value of the 

$\overline{\mathrm{K}}$. A pronounced dependence on the twist angle is discernible. Note that though the quasiparticle spectrum intensity at the top of the valence band is weak in the case of (d), (e) and (f), it was strong enough to allow for the determination of the maximum energy at $\bar{\Gamma}$.

Our $\mu$-ARPES measurements of twisted bilayer $\mathrm{MoS}_{2}$ along the high symmetry directions $\overline{\mathrm{M}}-\bar{\Gamma}-\overline{\mathrm{K}}$, and covering twist angles $\theta=0^{\circ}$ (AA stacking), $13.5^{\circ}, 26.5^{\circ}, 39^{\circ}, 47.5^{\circ}$ and $60^{\circ}$ (AB stacking, normal bilayer), are shown in Fig. 2 (c)-(h) as second-derivative-filtered topmost valence bands. The unfiltered bands over a larger energy range are shown in Fig. S3. (The $60^{\circ}$ data was taken from an earlier report ${ }^{3}$ ). The electronic structure of the topmost valence bands of $\mathrm{MoS}_{2}$ is derived from hybridization of the Mo $4 d$ and S 3p orbitals (see Fig. 2(a)) 19,20,21,22, 23, 24 , each of which possesses a strongly varying photonenergy-dependent photoionization cross-section ${ }^{25}$. In our measurements, a photon energy of $42 \mathrm{eV}$ was used. At this energy, the photoionization cross-section of the Mo $4 \mathrm{~d}$ subshell is an order of magnitude larger than that of S 3p. Thus, the primary contribution to our $\mu$-ARPES measurements, as shown in the bilayer $\mathrm{MoS}_{2}$ in Fig. 2(b), is from the Mo 4d orbitals. In our experiments, the incident photon flux was directed normal to the sample surface so that its polarization is in the plane of the $\mathrm{MoS}_{2}$ layers, thus suppressing excitation of states with out-of-plane character. This cross-sectional variation explains why the Mo- and S-derived states with a $z$ (or out-of-plane) component, i.e., $d_{z^{2}}$ or $p_{z}$, located in the uppermost valence band (UVB) near $\bar{\Gamma}$ (see Fig. 2(a)) have a consistently relatively weaker, but still nonzero, intensity. Note, also, that in our measurements, the ARPES signal is derived predominately from the topmost layer due to the mean-free-path of the corresponding inelastic photo-electrons is $<7 \AA$, which is shorter than a bilayer $\mathrm{MoS}_{2}$ thickness. Therefore, we were not able to measure significant signal intensity from states localized to the bottom layer.

Our ARPES data clearly show the relatively large change in the $\bar{\Gamma}$ state, as compared to the $\overline{\mathrm{K}}$ state, for several twist angles. To see this $\bar{\Gamma}$ state evolution more clearly, we used the second-derivative filtering method ${ }^{26}$ and referenced the bands with respect to the energy level of the state at $\overline{\mathrm{K}}$ (Fig. 2(c)(h)). Spin-orbit interactions lead to small band splitting at $\overline{\mathrm{K}}$ (as discernible in Fig. 2(a)), which is below our experimental resolution. The measured energy of the $\overline{\mathrm{K}}$ state and the hole effective mass at $\overline{\mathrm{K}}$ (see below) are therefore averaged values (for details see Supplementary section iii). It is clear from Fig. 2 (c)-(h) and Fig. S3 that for TB-MoS ${ }_{2}$ the VBM lies at $\bar{\Gamma}$ instead of $\overline{\mathrm{K}}$, contrary to $\mathrm{ML} \mathrm{MoS}_{2}$, but in agreement with earlier $\mathrm{ARPES}^{3,27,28}$ and photoluminescence ${ }^{11,12,13,14}$ reports. This band location corresponds to the indirect transition. As shown in Fig. 2(a), a change of the VBM at $\bar{\Gamma}$ between $30^{\circ}$ and $60^{\circ}$ is found in our theoretical calculation. The VBM at $\bar{\Gamma}$ shifts downward in energy as the twist angle is varied from $0^{\circ}$ or $60^{\circ}$ toward $\sim 40^{\circ}$. Note that in Fig. 2 (c)-(h), the red bars and arrows qualitatively denote the energy difference between $\bar{\Gamma}$ and $\overline{\mathrm{K}}$ valence band maxima; energy differences were determined via energy distribution curves (EDCs) peak fittings (see Supplementary Material Figure S6). These extracted values are further compared to the PL measurements and theoretical calculations in Fig. 3(b). The spectrum away from $\bar{\Gamma}$ is also evolving with twist angle. For instance, the relative energy positions of the $\overline{\mathrm{M}}$ point and of the local minimum labeled $\overline{\mathrm{J}}$ with respect to $\overline{\mathrm{K}}$, are also changing with twist angle. We comment further on this below. 

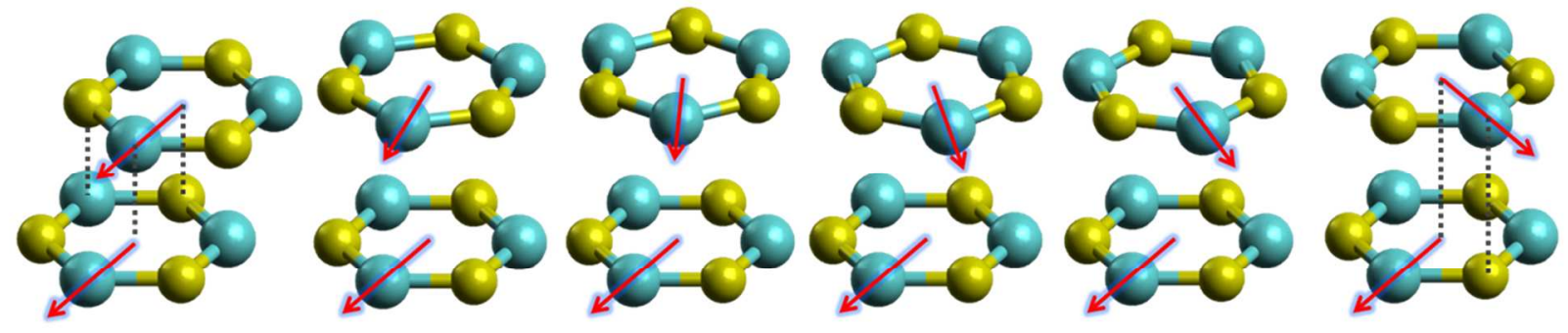

(b)

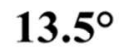

26.5

$39^{\circ}$

$47.5^{\circ}$

(c)
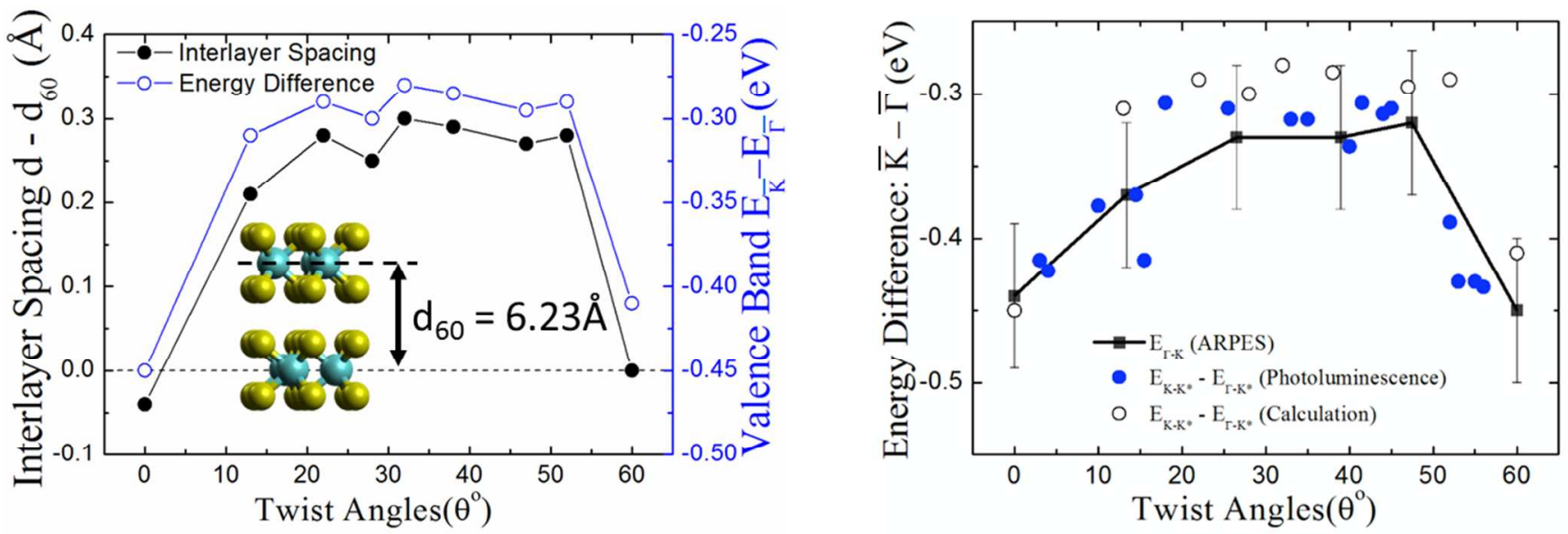

Fig. 3 (a) Schematics of relative orientation and stacking of the individual layers for each twist angle measured in this work. The arrows denote the orientation of each layer. (b) Calculated interlayer spacing and corresponding energy difference between VBM at $\bar{\Gamma}$ and $\overline{\mathrm{K}}$ versus twist angles, derived from DFT. The interlayer spacing is defined as the mean separation between the Mo-Mo or S-S layers, and is referenced with respect to the $60^{\circ}$ (normal bilayer $\mathrm{MoS}_{2}$ ). This result is adapted from [10]. (c) A comparison of the energy difference from our ARPES results (solid lined-squares), the calculation ${ }^{11}$ (empty circles), and the transition energy from PL measurement ${ }^{11}$ (blue solid circles). To allow for comparison, the ARPES and PL experimental data are referenced/aligned to the data point at $60^{\circ}$. The energies and error bars of the ARPES points are derived from the mean and standard deviation over the six high symmetry points of $\overline{\mathrm{K}}$ for each twist angle.

The origin of the bandgap opening with twist angle is the interlayer coupling that predominately affects the valence band states. According to theoretical predictions ${ }^{11,12,13,15,16}$, the relative orientation of the top and bottom layers of bilayer $\mathrm{MoS}_{2}$ leads to a change in the interlayer spacing which is proportional to the degree of interlayer coupling (see Fig. 3(a)\&(b)). The VBM at $\bar{\Gamma}$, derived from out-of-plane $S p_{z}$ and Mo $d_{z^{2}}$ orbitals (see Fig. 2(a)), is sensitive to the out-of-plane interlayer coupling. Increasing the twist angle of $\mathrm{TB}-\mathrm{MoS}_{2}$ from $0^{\circ}$ to $30-40^{\circ}$ leads to an increase in the interlayer spacing, and thus a decrease in the interlayer coupling, resulting in an overall downward shifting in energy of the $\bar{\Gamma}$ state by up to $120 \mathrm{meV}$. Since the states of the VBM at $\bar{K}$, mostly derived from Mo $d_{\mathrm{x}^{2}-\mathrm{y}^{2} / x y}$ orbitals, are effectively invariant to the range of interlayer spacings encountered here, as evidenced by their lack of any significant shifts (and the small changes in the direct-gap size as measured by $\mathrm{PL}^{11,13,28}$ ), the interlayer twist-induced bandgap shift is primarily determined by the 
relative energy position of the $\bar{\Gamma}$ state. This correlation between twist angle and both (1) the indirect optical excitation from previously reported PL measurements, and (2) the $\overline{\mathrm{K}}-\bar{\Gamma}$ energy difference from our ARPES measurements, is shown in Fig. 3(c). The evolution of the $\overline{\mathrm{K}}-\bar{\Gamma}$ energy difference vs. twist angle is clearly observed by our ARPES measurements, and agrees well with the PL data 11 and theoretical calculations 11. Note that the ARPES and PL data shown in Fig. 3(c) are aligned with respect to the $2 \mathrm{H}-\left(60^{\circ}\right)$ bilayer $\mathrm{MoS}_{2}$ and the error bars denote the standard deviation of fits for all six equivalent high-symmetry directions. While PL measures optical excitations that include excitonic effects, DFT and ARPES provide single-particle energies. A comparison, as shown in Fig. 3(c), is possible under the assumption that the binding energies of the excitons do not change with twisting; for in this case, a change of the band gap will rigidly translate to a change of the PL frequency. The comparison between ARPES, DFT, and photoluminescence results provides support for this assumption of a twistangle independent exciton binding energy. Thus, our results provide direct experimental evidence for the predominant mechanism controlling the change in the bandgap in a bilayer $\mathrm{MoS}_{2}$ system by interlayer twist angle.

In addition to the evolution of the $\bar{\Gamma} / \overline{\mathrm{K}}$ states with twist angle, our measurements also show the evolution of the entire valence band structure with interlayer twist. By following this more detailed electronic structure evolution, we are led to a more comprehensive picture of the twist-angle dependent interlayer coupling. This, for example, can be seen in the valence band evolution of the $\overline{\mathrm{K}}-\overline{\mathrm{M}}$ and $\overline{\mathrm{K}}-\overline{\mathrm{J}}$ energy differences in Fig. 4 (a). Furthermore, unlike the states near $\bar{\Gamma}$, note that there is no clear indication of significant bi-layer induced splitting in states near $\bar{M}$ or $\bar{J}$ in our ARPES measurements nor in DFT (Fig. 2 (a)) (the splitting in $\bar{J}$ is due to spin-orbit interactions); this indicates that these states are localized to the respective layer of the twisted-bilayer (further supported by the orbital characters of $\bar{M}$ and $\bar{J}$ in Fig. 2(a)) and that their measured level shifts are different from the effects of interlayer hybridization discussed above. This result suggests a non-trivial role of the atomic registry. Note that we can rule out the possible role of spurious doping since the change in energy position of the state at $\overline{\mathrm{K}}$ with respect to the Fermi level, an indicator of doping, does not show the same trend; in other words, the trend in doping with twist angle is uncorrelated to the trend of the $\overline{\mathrm{K}}$ $\overline{\mathrm{M}}, \overline{\mathrm{K}}-\overline{\mathrm{J}}$, and $\overline{\mathrm{K}}-\bar{\Gamma}$ energy differences (see Fig 4(a) and Fig. S4). On the other hand, the trends of $\overline{\mathrm{K}}-\overline{\mathrm{M}}$ and $\overline{\mathrm{K}}-\mathrm{J}$ are clearly correlated, as shown in Fig. 4(a), and their trend does show some similarities with that of (negative) $\overline{\mathrm{K}}-\bar{\Gamma}$, shown in Fig $3(\mathrm{c})$, indicating that their evolution is due to the relative interlayer orientation. Thus, while the energy of the valence band edge (at the $\bar{\Gamma}$ point) is mostly determined by the interlayer hybridization, we find that the evolution of the electronic structure away from $\bar{\Gamma}$ is related to other reasons (see supplementary section for further analysis). This has ramifications for the electronic properties of TB-MoS , as in the case of the hole effective mass discussed below.

Hole Effective Mass $\left(m_{h}\right)$ vs Twist Angle $\left(\theta^{\circ}\right)$

\begin{tabular}{l|l|l|l|l|l|l}
\hline & $0^{\circ}$ & $13.5^{\circ}$ & $26.5^{\circ}$ & $39^{\circ}$ & $47.5^{\circ}$ & $60^{\circ}$ \\
\hline At $\bar{K}$ & $0.78 \pm 0.10$ & $1.23 \pm 0.16$ & $1.30 \pm 0.17$ & $1.13 \pm 0.15$ & $0.88 \pm 0.12$ & $1.06 \pm 0.15$ \\
\hline
\end{tabular}

Table 1 Hole effective mass in units of electron mass $m_{e}$, extracted from ARPES measured band structure at $\overline{\mathrm{K}}$ for several twist angles. 
(a)

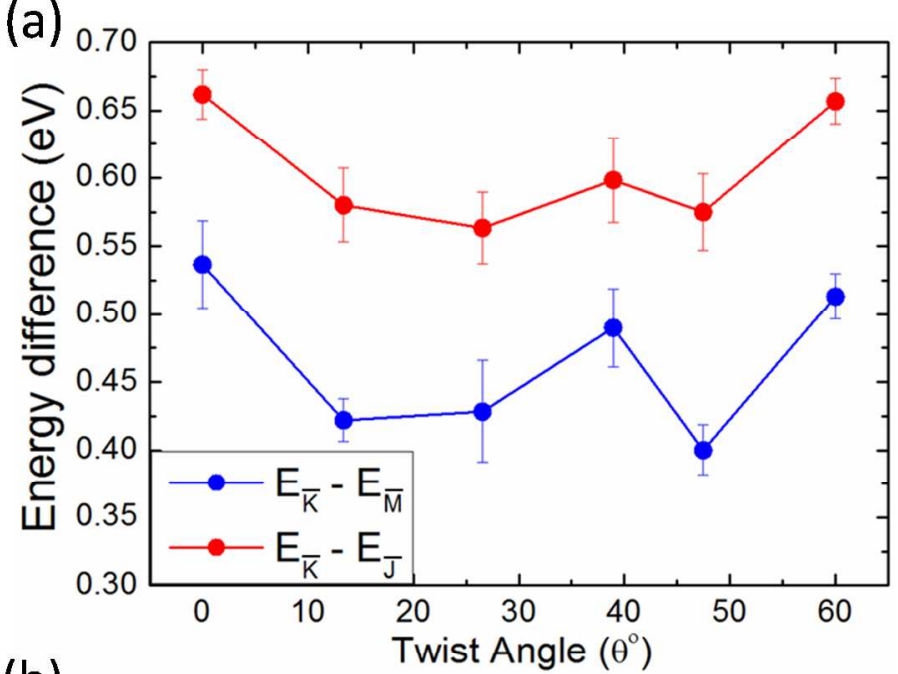

(b)

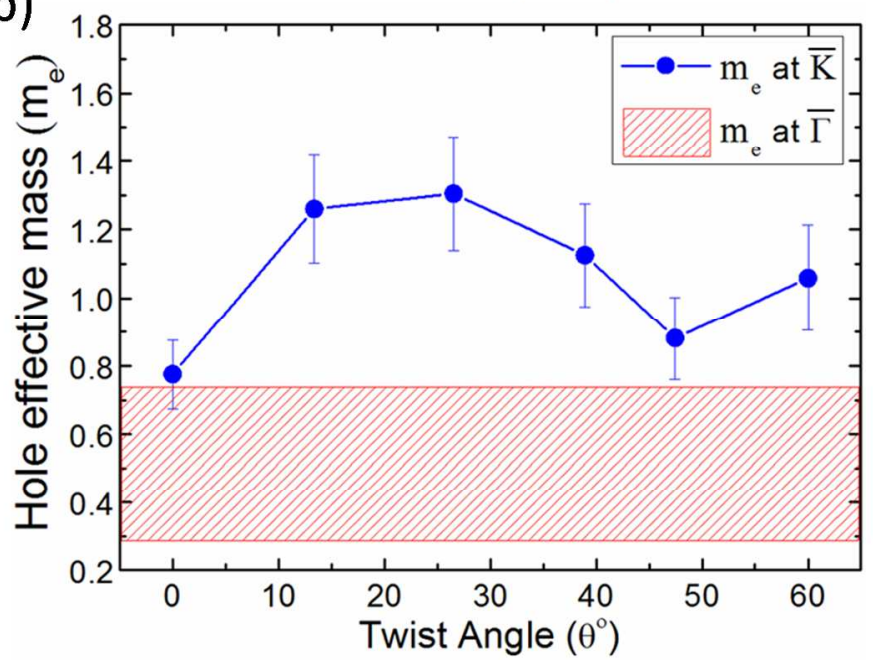

Fig. 4 (a) Energy differences between the VBM at the $(\overline{\mathrm{K}}, \overline{\mathrm{M}})$ and $(\overline{\mathrm{K}}, \overline{\mathrm{J}})$ points for different twist angles. Note the similar trends in twist-angle dependence. (b) (Blue line) Plot of the hole effective mass vs. twist angle at $\overline{\mathrm{K}}$. The red band denotes the approximate range of the hole effective mass at $\bar{\Gamma}$ over all twist angles.

An analysis of the curvature of the bands from the $\mu$-ARPES measurements allows us to deduce the effective mass of our TB-MoS 2 layers. This analysis takes into account the spectral weight of the bands including that arising from spin-orbit coupling. In particular, an analysis of the orbital composition of the states near the valence band maximum at the $\overline{\mathrm{K}}$ point reveals that the spectral weight is nearly identical in all four bands, i.e., the VBM (two-fold degenerate in 60 twisted bilayers) as well as the split-off bands (also two-fold degenerate). Since the energy difference between the two VBM bands is smaller than our energy resolution, and their nearly parallel dispersion (1.1\% difference) in the vicinity of $\overline{\mathrm{K}}$, the measured effective mass is an accurate representation of the mass for either band. Figure 4 (b) shows the evolution of the hole effective mass at $\overline{\mathrm{K}}$ for each measured twist angle; the corresponding values are also shown in Table 1 . The hole effective mass at $\bar{K}$ has a significant variation of up to $\sim 45 \%$ between $\theta=0^{\circ}$ and $26.5^{\circ}$ and it is generally larger than that predicted by DFT 
calculations (see Table S1 and Ref. 12) for crystallographic twist angles $0^{\circ}(0.78 \pm 0.10$ vs 0.582$)$ and $\left.60^{\circ}(1.06 \pm 0.12 \text { vs } 0.577)^{12}\right)$; the discrepancy is more significant when reaching $30^{\circ}\left(1.30 \pm 0.17\right.$ at $26.5^{\circ}$ vs. 0.44 at $30^{\circ 12}$ ). This evolution of the effective mass at $\overline{\mathrm{K}}$ with twist-angle is almost (negatively-) correlated with the evolution of the relative energy position of $\bar{J}$ with respect to $\overline{\mathrm{K}}$; in turn, the evolution of the relative position of $\bar{J}$ is not fully captured by DFT, and hence neither is the evolution of the effective mass $\overline{\mathrm{K}}$. While finite energy resolution, slight asymmetry along each high symmetry direction, and homogeneous in-plane strain (see Supplementary Information) does contribute to the error, it is also apparent that there is an overall difference in band dispersion between theory and experiment. We note that a "renormalization" of the bands of other similar 2D dichalcogenides has also been reported ${ }^{29,30,31}$. Due to the weak intensity of the measured bands near $\bar{\Gamma}$ in our normalincidence ARPES configuration, an accurate value for the effective mass at $\bar{\Gamma}$ cannot be extracted; however, our measurements do allow us to estimate a range of $(0.41 \pm 0.13) m_{0}$ to $(0.65 \pm 0.09) m_{0}$ (where $m_{0}$ is the electron mass) for the hole effective mass at $\bar{\Gamma}$ over the twist angle range of $0^{\circ}$ to $60^{\circ}$, as denoted by the red-hatched band in Fig. 4(b). The corresponding DFT values (see Table S1) are significantly larger and range from $1.1 \mathrm{~m}_{0}\left(\right.$ for $0^{\circ}, 60^{\circ}$ ) to $1.5 \mathrm{~m}_{0}\left(\right.$ at $\left.30^{\circ}\right)$. This result is surprising as one would assume that the effective mass at $\bar{\Gamma}$ should change more with twist angle than that at $\overline{\mathrm{K}}$ because the band energy of the former varies the strongest with twist. Our results show that the opposite is the case because the upshift of the $\bar{J}$ point energy with twist reduces the band curvature at $\overline{\mathrm{K}}$ and therefore increases its effective mass.

In conclusion, we have studied the occupied electronic bands of CVD-grown, and then drytransferred bilayer $\mathrm{MoS}_{2}$ with different twist angles ranging from $0^{\circ}$ to $60^{\circ}$. Bright field LEEM provided real-space structural measurements of TB-MoS 2 , while dark-field LEEM imaging, combined with $\mu$-LEED, was used to define the interlayer crystal orientations. Our $\mu$-ARPES measurements have probed the full Brillouin zone of the valence band structure of bilayer $\mathrm{MoS}_{2}$ at twist angles of $0^{\circ}, 13.5^{\circ}, 26.5^{\circ}, 39^{\circ}$, $47.5^{\circ}$, and $60^{\circ}$. We observed an energy shift of up to $120 \mathrm{meV}$ at the VBM at $\bar{\Gamma}$ when the twist angle increases from $0^{\circ}$ to $40^{\circ}$. Since the relative energy separation of the VBM and CBM at $\bar{K}$ was found to be weakly dependent on interlayer twist, as confirmed by PL measurements, the bandgap opening is determined predominately by the energy-shift of the VBM at $\bar{\Gamma}$ with varying twist angle. This variation at $\bar{\Gamma}$, which agrees with previously reported PL measurements and DFT calculations, is mostly due to the evolution of the interlayer coupling strength, which in turn is a function of the interlayer spacing. Thus, the $\bar{\Gamma}$ VBM state shifts in energy with twist angle, and thus the relative orientation of the two layers can be used to tune the bandgap of bilayer $\mathrm{MoS}_{2}$. In addition, our measurements over the entire Brillouin zone reveal other variations in the band structure that are indicative of more complicated physics at play. One consequence of this electronic structure variation is the unusual evolution of the effective mass with twist angle. We expect that these results will provide physical insight into the optical and electronic properties of TB-MoS 2 , and to controlling the bandgap, transport, and spin-/valley properties by tuning the interlayer coupling, since the ability to control the interlayer coupling is important to the development of TMD-based devices. 


\author{
ASSOCIATED CONTENT \\ Supporting Information \\ Detailed descriptions of: \\ i. Sample preparation \\ ii. LEEM and ARPES Measurements \\ iii. Further discussion and analysis of energy differences \\ iv. Effective mass \\ v. Determination of energy difference between $\Gamma$ and $K$ using EDCs peak fitting \\ vi. Details of the density functional theory calculations
}

\title{
AUTHOR INFORMATION
}

\section{Corresponding Author}

*E-mail: py2175@Columbia.edu.

\section{Notes}

The authors declare no competing financial interest.

\section{ACKNOWLEDGEMENT}

The beamline measurements and analyses and the sample mounting were supported by the Department of Energy, Office of Basic Energy Sciences, Division of Materials Sciences and Engineering under Award Contract No. DE-FG 02-04-ER-46157. This research used resources of the Center for Functional Nanomaterials and National Synchrotron Light Source, which are U.S. DOE Office of Science User Facilities, at Brookhaven National Laboratory under Contract No. DE-SC0012704 (J.S. and P.S.). The sample preparation and optical characterization (by D.C., A.G., and J.H.) and twist theory (J.K.) were supported as part of the Center for Redefining Photovoltaic Efficiency through Molecular Scale Control, an Energy Frontier Research Center (EFRC) funded by the U.S. Department of Energy (DOE), Office of Science, Basic Energy Sciences under Award No. DE-SC0001085. The EFRC work is also supported by a matching grant from the Empire State Development's Division of Science, Technology and Innovation (NYSTAR), Contract \# C090147, as well as by the New York State Energy Research Development Authority (NYSERDA), Contract \# 17353.

\section{ABBREVIATIONS \\ Molybdenum-disulfide $\left(\mathrm{MoS}_{2}\right)$, transition-metal dichalcogenides (TMDs), photoluminescence (PL), chemical-vapor-deposition (CVD), valence band maximum (VBM), conduction band minimum (CBM), angle resolved photoemission (ARPES), twisted bilayer $\mathrm{MoS}_{2}$ (TB-MoS $)$, micro-spot angle-resolved photoemission spectroscopy ( $\mu$-ARPES), bright-field (BF), dark-field (DF), low-energy electron microscopy (LEEM), micro-spot low-energy electron diffraction ( $\mu$-LEED).}

\section{REFERENCES}


1. Splendiani, A.; Sun, L.; Zhang, Y.; Li, T.; Kim, J.; Chim, C. Y.; Galli, G.; Wang, F. Nano Letters 2010, 10(4), 1271-1275.

2. Conley, H. J.; Wang, B.; Ziegler, J. I.; Haglund Jr, R. F.; Pantelides, S. T.; Bolotin, K. I. Nano Letters 2013, 13(8), 3626-3630.

3. Jin, W.; Yeh, P.-C.; Zaki, N.; Zhang, D.; Sadowski, J. T.; Al-Mahboob, A.; van Der Zande, A. M.; Chenet, D. A.; Dadap, J. I.; Herman, I. P.; Sutter, P.; Hone J.; Osgood Jr., R. M. Physical Review Letters 2013, 111(10), 106801.

4. Yeh, P.-C.; Jin, W.; Zaki, N.; Zhang, D.; Liou, J. T.; Sadowski, J. T.; Al-Mahboob A.; Dadap, J.I.; Herman, I.P.; Sutter, P. and Osgood Jr, R.M., Physical Review B 2015, 91(4), 041407.

5. Kośmider, K.; Fernández-Rossier, J. Physical Review B 2013, 87(7), 075451.

6. Jin, W.; Yeh, P.-C.; Zaki, N.; Chenet, D.; Arefe, G.; Hao, Y.; Sala, A.; Mentes, T.O.; Dadap, J.I.; Locatelli, A.; Hone, J.; Osgood Jr. R. M. Physical Review B 2015, 92(20), 201409.

7. Mak, K. F.; Lee, C.; Hone, J.; Shan, J.; Heinz, T. F. Physical Review Letters 2010, 105(13), 136805.

8. Lee, C.; Yan, H.; Brus, L. E.; Heinz, T. F.; Hone, J.; Ryu, S. ACS Nano 2010, 4(5), 2695-2700.

9. Wu, S.; Ross, J. S.; Liu, G. B.; Aivazian, G.; Jones, A.; Fei, Z.; Zhu W.; Xiao, D.; Yao, W.; Cobden, D.; Xu, X. Nature Physics 2013, 9(3), 149-153.

10. Y Yuan, H.; Bahramy, M.S.; Morimoto, K.; Wu, S.; Nomura, K.; Yang, B.J.; Shimotani, H.; Suzuki, R.; Toh, M.; Kloc, C.; Xu, X. Nature Physics 2013, 9(9), 563-569.

11. van Der Zande, A.M.; Kunstmann, J.; Chernikov, A.; Chenet, D.A.; You, Y.; Zhang, X.; Huang, P.Y.; Berkelbach, T.C.; Wang, L.; Zhang, F.; Hybertsen, M.S. Nano Letters 2014, 14(7), 3869-3875.

12. Huang, S.; Ling, X.; Liang, L.; Kong, J.; Terrones, H.; Meunier, V.; Dresselhaus, M.S. Nano Letters 2014, 14(10) 5500-5508.

13. Liu, K.; Zhang, L.; Cao, T.; Jin, C.; Qiu, D.; Zhou, Q.; Zettl, A.; Yang, P.; Louie, S.G.; Wang, F. Nature Communications 2014, 5, 4966.

14. Castellanos-Gomez, A.; van der Zant, H. S. J.; Steele, G. A. Nano Research 2014, 7(4), 572-578.

15. Cao, B.; Li, T. The Journal of Physical Chemistry C 2015, 119(2), 1247-1252.

16. Wang, Z.; Chen, Q.; Wang, J. The Journal of Physical Chemistry C 2015, 119(9), 4752-4758.

17. Locatelli, A.; Knox, K.R.; Cvetko, D.; Mentes, T.O.; Nino, M.A.; Wang, S.; Yilmaz, M.B.; Kim, P.; Osgood Jr, R.M.; Morgante, A. ACS Nano 2010, 4 (8), 4879-4889.

18. Feenstra, R.M.; Srivastava, N.; Gao, Q.; Widom, M.; Diaconescu, B.; Ohta, T.; Kellogg, G.L.; Robinson, J.T.; Vlassiouk, I.V. Physical Review B 2013, 87(4), 041406.

19. Cappelluti, E.; Roldán, R.; Silva-Guillén, J. A.; Ordejón, P.; Guinea, F. Physical Review B 2013, 88(7) 075409.

20. Liu, G. B.; Shan, W. Y.; Yao, Y.; Yao, W.; Xiao, D. Physical Review B 2013, 88(8), 085433.

21. Zhu, Z. Y.; Cheng, Y. C.; Schwingenschlögl, U. Physical Review B 2011, 84(15), 153402.

22. Mattheiss, L. F. Physical Review B 1973, 8(8), 3719.

23. Coehoorn, R.; Haas, C.; Dijkstra, J.; Flipse, C. J. F.; De Groot, R. A.; Wold, A. Physical Review B 1987, 35(12), 6195.

24. Chang, C. H.; Fan, X.; Lin, S. H.; Kuo, J. L. Physical Review B 2013, 88(19), 195420.

25. Yeh, J. J.; Lindau, I. Atomic Data and Nuclear Data Tables 1985, 32(1), 1-155.

26. Zhang, P.; Richard, P.; Qian, T.; Xu, Y. M.; Dai, X.; Ding, H. Review of Scientific Instruments 2011, 82(4), 043712. 
27. Eknapakul, T.; King, P.D.C.; Asakawa, M.; Buaphet, P.; He, R.H.; Mo, S.K.; Takagi, H.; Shen, K.M.; Baumberger, F.; Sasagawa, T., Jungthawan, S. Nano Letters 2014, 14(3), 1312-1316.

28. Latzke, D.; Zhang, W.; Tongay, S.; Chang, T.R.; Lin, H.; Jeng, H.T.; Suslu, A.; Wu, J.; Bansil, A.; Lanzara, A. Physical Review B 2015, 91(23), 235202.

29. Ugeda, M.M.; Bradley, A.J.; Shi, S.F.; Felipe, H.; Zhang, Y.; Qiu, D.Y.; Ruan, W.; Mo, S.K.; Hussain, Z.; Shen, Z.X.; Wang, F. Nature Materials 2014, 13(12), 1091-1095.

30. Jin, W.; Yeh, P.C.; Zaki, N.; Zhang, D.; Liou, J.T.; Sadowski, J.T.; Barinov, A.; Yablonskikh, M.; Dadap, J.I.; Sutter, P.; Herman, I.P.; Osgood Jr., R. M. Physical Review B 2015, 91(12), 121409.

31. Zhang, Y.; Chang, T.R.; Zhou, B.; Cui, Y.T.; Yan, H.; Liu, Z.; Schmitt, F.; Lee, J.; Moore, R.; Chen, Y.; Lin, H. Nature Nanotechnology 2014, 9(2), 111-115. 